\title{
Вопросы построения и применения базы знаний в проектировании и производстве инновационных объектов
}

\author{
Р.И. Сольнииев 1, д.m.н., nрофрессор, remira70@mail.ru
}

Г.И. Коршунов 2, д.m.н., npoфpeccop,kgi@pantes.ru

До Суан Чо ${ }^{3}$, к.m.н., chodx@ptit.edu.vn

До Хай Куан 1, магистрант, quan221212@gmail.com

${ }^{1}$ Санкт-Петербургский государственный электротехнический университет "АЭТИ" им. В.И. Ульянова (Иенина), г. Санкт-Петербург, 197376, Россия

2 Институт аэрокоспических приборов и систем (ГУАП),

2. Санкт-Петербург, 190000, Россия

3 Почтово-телекоммуникаиионный технологический институт, г. Ханой, Въетнам

В статье рассматриваются принципы построения и применения базы знаний для инновационных объектов. Описываются формирование и применение базы знаний при проектировании и производстве на примере замкнутой системы управления нейтрализацией выхлопных газов автотранспорта, являющейся инновационным объектом в области функционирования системы управления «природа-техногеника». Обоснован выбор замкнутой системы управления нейтрализацией выхлопных газов автотранспорта в качестве эталона инновационных объектов проектирования и производства. Сквозной цифровой процесс проектирования и производства этого объекта строится на основе базы знаний и включает разработку принципиальных и электрических схем, конструкторских решений и документации, ПО, а также изготовление, сборку и настройку прототипов.

В базу знаний вводятся необходимные для проектирования и производства рассматриваемого объекта данные и знания. Так, для этапа схемотехнического проектирования вводится электрическая схема замкнутой системы управления нейтрализацией выхлопных газов автотранспорта, разрабатываемая инструментами проектирования PCAD в соответствии с хранящимися в базе знаний установленными стандартами. Средства САПР и автоматизированных систем технологической подготовки производства для дальнейших процедур обеспечивают учет стандартов IPC также через базу знаний, в том числе разработку конструкторских решений и документации на изготовление печатных плат и других элементов. На основе базы знаний выполняется и разработка проектной документации на последующих этапах проектирования. При этом хранящиеся в ней фрагменты конструкции используются для новых конструкторских решений и изготовления конструкторской документации.

Предложена система управления базой знаний, позволяющая с помощью нотации UML выполнять ее поддержку разработчиками, получать ответы на запросы рядовых инженеров-проектировщиков (схемотехников, конструкторов, технологов, испытателей), обеспечивать ввод и контроль знаний экспертами.

Приводится интерфейс пользователя, в котором представляются поля ввода запросов и индикации ответов, а также операторы управления и визуализации. На примерах показано применение разработанной базы знаний для процедур схемотехнического и конструкторского этапов проектирования и технологической подготовки производства.

Применение базы знаний для конкретных проектных и производственных процедур осуществляется на основе проблемно-ориентированных языков пользователей при формировании запросов и ответов. Полученные результаты распространяются на широкий класс инновационных объектов.

Ключевые слова: замкнутая система управления, инновачионные объекты, база знаний, система управления базой знаний, каталитический нейтрализатор, проектирование и производство.

В работе рассматривается формирование базы знаний (БЗ) и системы управления ею с привязкой к соответствующим процедурам схемотехнического, конструкторского, технологического и испытательного этапов проектирования и подготовки производства на при- мере инновационного объекта - замкнутой $\mathrm{cu}$ стемы управления нейтрализачией выхлопных газов автотранспорта (ЗСУ НВГА).

Различные подходы к построению Б3 [1-5], как правило, не привязываются к конкретным процедурам проектирования и производства. 
В то же время на каждом этапе специалисты в конкретной области должны иметь инструментарий для запросов и ответов в повышенной динамике выполнения проектных и производственных процедур инновационных объектов. Статья является продолжением и развитием работ авторов по рассматриваемому направлению $[6,7]$.

\section{Объект проектирования}

Объектом проектирования, для которого необходимо построение Б3, в данном случае является ЗСУ НВГА, представленная на рисунке 1 в виде функциональной схемы (контроллеры 1-3 предназначены для индикации, управления теплонагревателем и приемопередачи ГЛОНАCC/GPS соответственно).

На рисунке 2 приведена блок-схема 3СУ НВГА для формирования блоков и элементов этих блоков как исходных сведений в БЗ.

\section{Процесс проектирования и подготовки производства}

Процесс проектирования и подготовки производства для ЗСУ НВГА включает разработку принципиальных и электрических схем, конструкторских решений и документации (в том

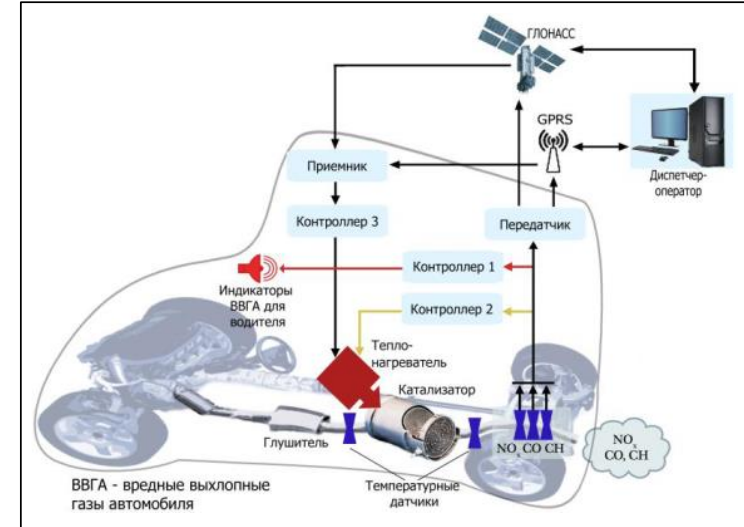

Рис. 1. Функциональная схема ЗСУ НВГА

Fig. 1. Functional diagram of CLC for NEGT

числе по печатным платам), ПО, а также изготовление, сборку и настройку прототипов.

Сквозной цифровой процесс проектирования и производства рассматриваемого объекта строится на основе БЗ, в которую вводятся необходимые для этого данные и знания. Электрическая схема ЗСУ НВГА разрабатывается инструментами проектирования PCAD в соответствии с хранящимися в Б3 установленными стандартами. Средства САПР и автоматизированных систем технологической подготовки производства (Симатрон, DFM и др.) для дальнейших процедур обеспечивают учет стандар-

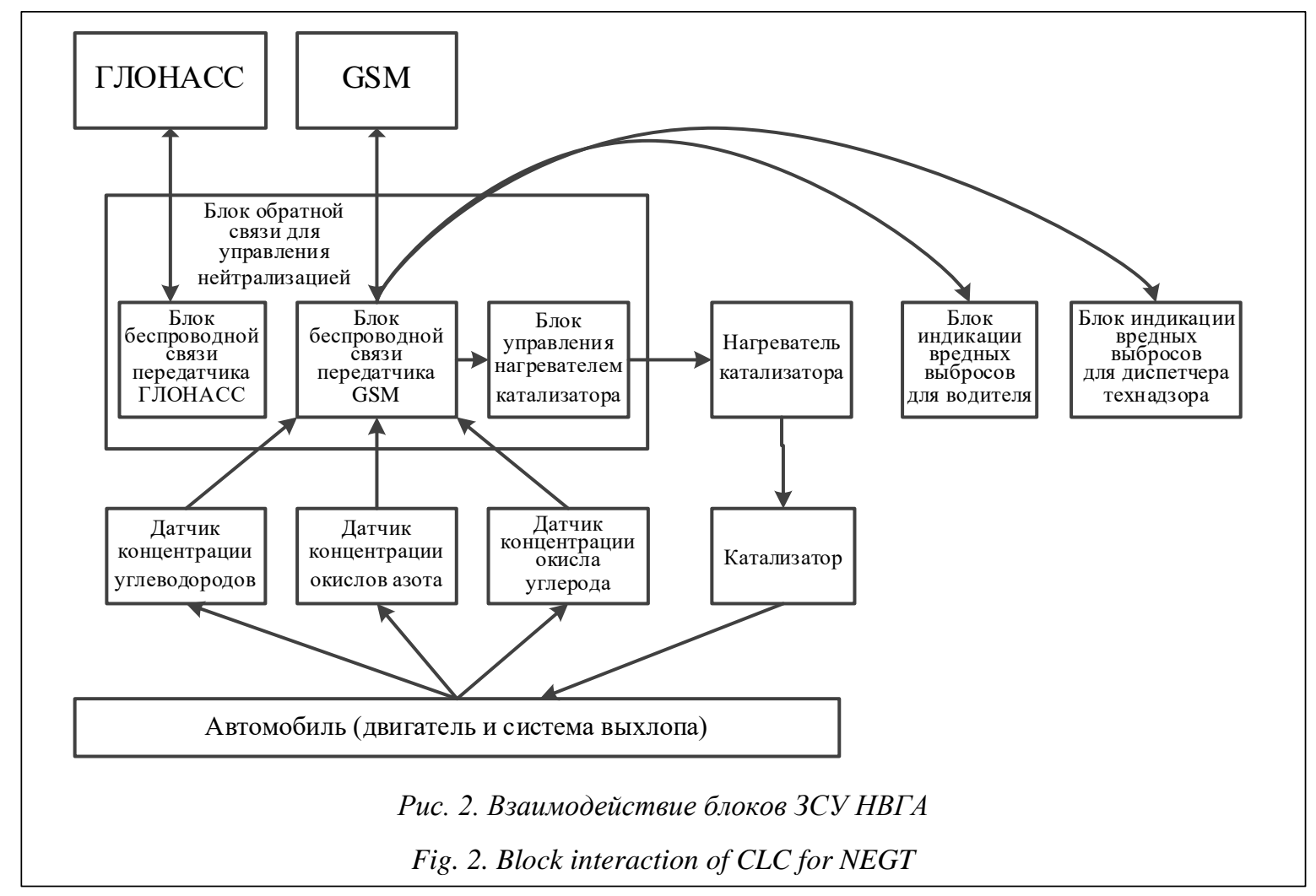


тов IPC через Б3, а также разработку конструкторских решений и документации на изготовление печатных плат и других элементов, проверку по PCBA (Printed Circuit Board Assembly) $[8]$.

Разработка проектной документации на последующих этапах проектирования также осуществляется на основе БЗ. При этом хранимые в Б3 фрагменты конструкции используются для новых конструкторских решений и изготовления конструкторской документации.

Одно из новых конструкторских решений показано на рисунке 3. Полученная версия размещения катализатора за резонатором в результате применения из БЗ фрагментов конструкторской разработки звена ЗСУ НВГА, а датчиков ЗСУ НВГА в багажнике позволяет поддерживать существующую конструкцию без значительных изменений.

\section{Формирование структуры Б3}

Б3 должна содержать нормативы, справочные данные, типовые проектные решения, закономерности и правила, необходимые для проектирования и производства инновационных объектов со специфичными для них динамичными процессами.

В представленной ЗСУ НВГА от БЗ требуются знания по следующим составляющим:

- датчики, преобразователи, элементы выхлопной системы автомобилей, катализаторы, теплонагреватели;

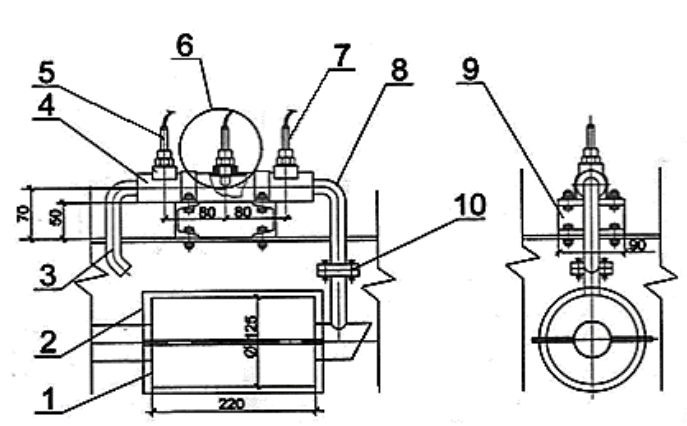

Рис. 3. Фрагмент конструкиии ЗСУ НВГА:

1 - катализатор с нагревательным элементом, 2 - корпус катализатора, 3 - наружная труба, 4 - коллектор, 5 - датчик $\mathrm{CH}, 6$ - датчик $\mathrm{CO}, 7$ - датчик NO, 8 - вход для труб, 9 - пьлеуловитель, 10 -монтажный комплект

Fig. 3. Fragment of construction of CLC for $E$ NEGT
- методики, алгоритмы анализа, синтеза и конструирования;

- требования и ограничения в процессе проектирования и производства, прецеденты, оценки экспертов по проектированию, связи процедур проектирования и т.д.;

- понятия, классы, свойства и атрибуты при проектировании и производстве ЗСУ НВГА, в том числе проектные процедуры, операции и другие связанные понятия;

- взаимосвязи основных концептов, используемых в проектировании ЗСУ НВГА.

Описываемая БЗ должна обладать необходимыми правилами логического вывода для принятия решений на всех этапах проектирования и подготовки производства ЗСУ НВГА, для выбора стратегии проектирования, а также для извлечения знаний с целью их учета при проектировании ЗСУ НВГА.

Кроме того, в БЗ должен присутствовать набор необходимых запросов для извлечения знаний при выполнении проектных и производственных процедур ЗСУ НВГА.

Выбор способа представления знаний проводился на основе функционального моделирования процессов формирования БЗ ЗСУ НВГА. В качестве метода такого моделирования выбран SADT с графической нотацией IDEF0, DFD [9-11].

На рисунке 4 представлен процесс формирования БЗ ЗСУ НВГА в нотации IDEF0. Входными данными для него являются концепции, элементы и модели системы, алгоритмы управления катализатором, конструкторские решения, выходными - разработанная Б3 (БД по элементам ЗСУ НВГА, БД по инновационным и стандартным конструкторским решениям ЗСУ НВГА и т.д.). Все пользователи делятся на категории: рядовые проектировщики, которым нужна информационная поддержка от Б3 (инженеры-разработчики); инженеры-конструкторы, инженеры-технологи, инженеры-испытатели, высококвалифицированные специалисты-эксперты; разработчики БЗ, являющиеся основным звеном по ее созданию (рис. 4 и 5).

Контекстная диаграмма процесса формирования БЗ проектирования ЗСУ НВГА (рис. 5) представлена в виде схемы потоков данных с позиций нотации DFD с декомпозицией на процессы создания Б3 и разработки системы управления БЗ (СУБЗ).

Формирование Б3 и СУБЗ выполняется разработчиками. СУБЗ ориентирована на рядового пользователя. Сопровождение информа- 
ционного и программного обеспечения осуществляется экспертами. При этом происходит связь как с разработчиками, так и с пользовате- лями БЗ, что позволяет выполнить экспертную оценку результатов проектирования и разработки с учетом механизма обратной связи с

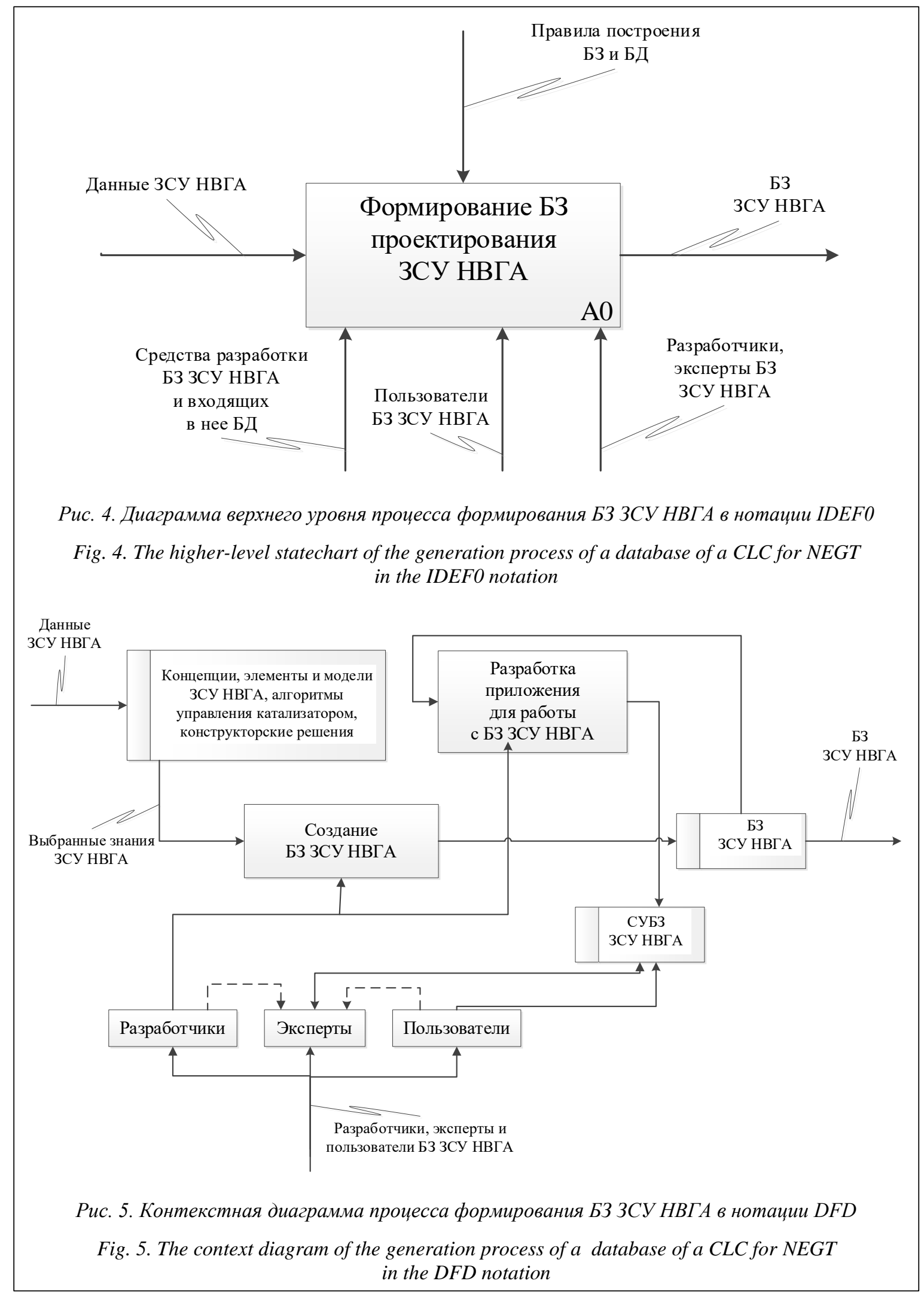


пользователями. Ключевая роль в предоставлении дополнений и коррекций лежит на экспертах. Роль каждого участника работы с Б3 с помощью нотации UML представлена на рисунке (см. http://www.swsys.ru/uploaded/image/20204/2020-4-dop/5.jpg).

Процесс формирования БЗ ЗСУ НВГА начинается со сбора информации, относящейся к предметной области знаний системы. Разработчики Б3 на этом этапе могут быть экспертами в рассматриваемой предметной области и иметь возможность общаться с настоящими экспертами для исключения противоречий и неточностей.

Входные знания для заполнения в Б3 ЗСУ НВГА формируются на основе концептуальных и конструктивных знаний, а также БД, содержащих информацию об элементах, входящих в систему. Поэтому можно считать, что Б3 ЗСУ НВГА включает в себя БЗ по концепциям, связанным с ЗСУ НВГА, БД элементов ЗСУ НВГА и БЗ по инновационным и стандартным проектно-производственным решениям ЗСУ НВГА.

Для каждой указанной предметной области проводится разделение на подобласти.

Подобласти БЗ по концепциям и схемотехническому этапу проектирования ЗСУ НВГА включают структуру ЗСУ НВГА, математические модели, методы математического моделирования и законы управления катализатором.

БД элементов системы включает данные микроконтроллеров, модулей ГЛОНАСС, датчиков ВГА, катализаторов, датчиков температуры, индикаторов ВГА, токсичных веществ.

Подобласти Б3 по инновационным и нестандартным схемотехническим решениям ЗСУ НВГА представлены на рисунке 6.

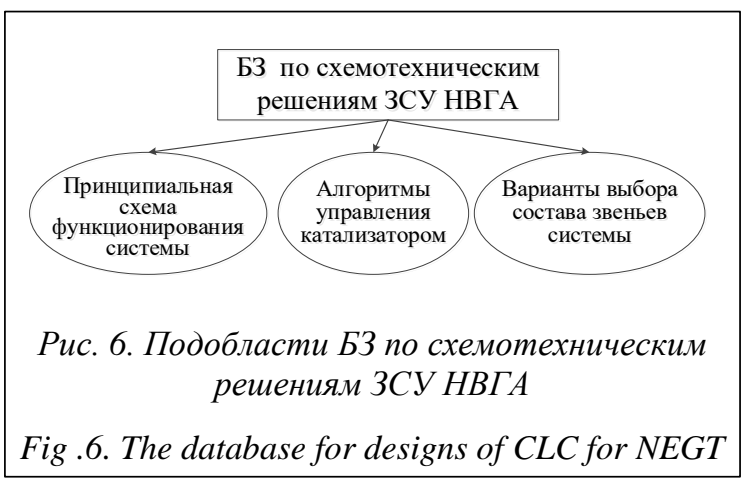

Аналогично строятся структуры для конструкторов, технологов и испытателей.

В соответствии с онтологическим подходом [9, 12-14] в структуре Б3 разделяются понятия, классы, слоты и определяются отношения между ними, а также выделяются экземпляры, из которых выбираются требуемые входные формы для запросов БЗ ЗСУ НВГА.

\section{Разработка СУБ3}

Предлагаемая СУБЗ предназначена для формирования и вывода ответов из БЗ на запросы пользователя в предметных областях ЗСУ НВГА. Эти ответы представляются текстовой и графической информацией. На рисунке 7 отражена работа СУБЗ с помощью нотаций UML.

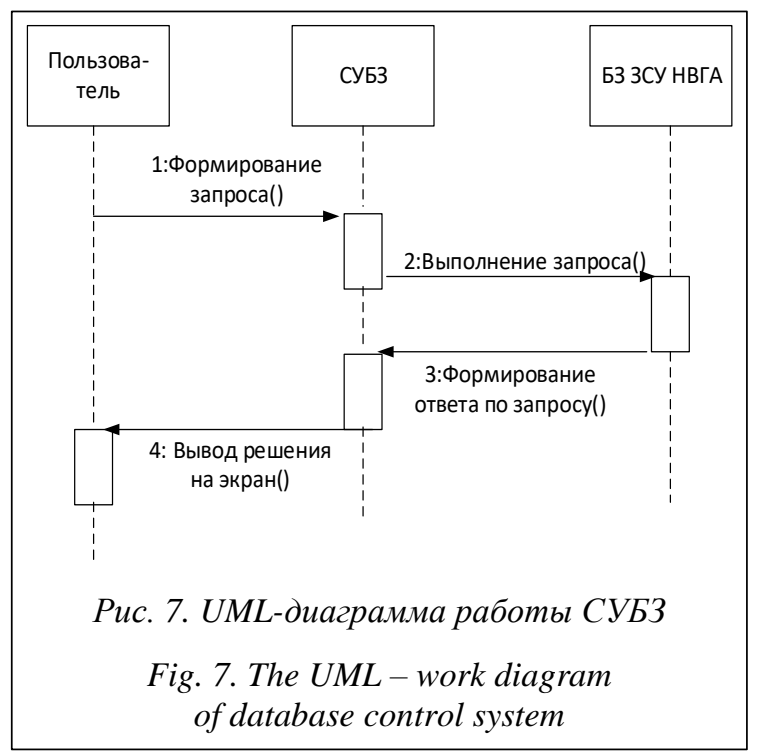

При работе с СУБЗ выполняются следующие действия:

- разработчики обеспечивают поддержку Б3, рассматривают предложения о дополнении/внесении информации от пользователей, вносят информацию, поступающую от экспертов;

- пользователи (рядовые проектировщики) получают информацию по запросу, могут вносить предложения по исправлению информации, которые далее рассматриваются разработчиками;

- эксперты или опытные специалисты вводят новые знания и взаимосвязи между ними.

С помощью созданной СУБЗ пользователь на проблемно-ориентированном языке сможет осуществлять запросы, которые относятся к конкретным процедурам на этапах схемотехнического проектирования, конструкторской разработки и прочего, и получать ответ из БЗ на данный запрос в виде текстовых файлов типа *.doc. 


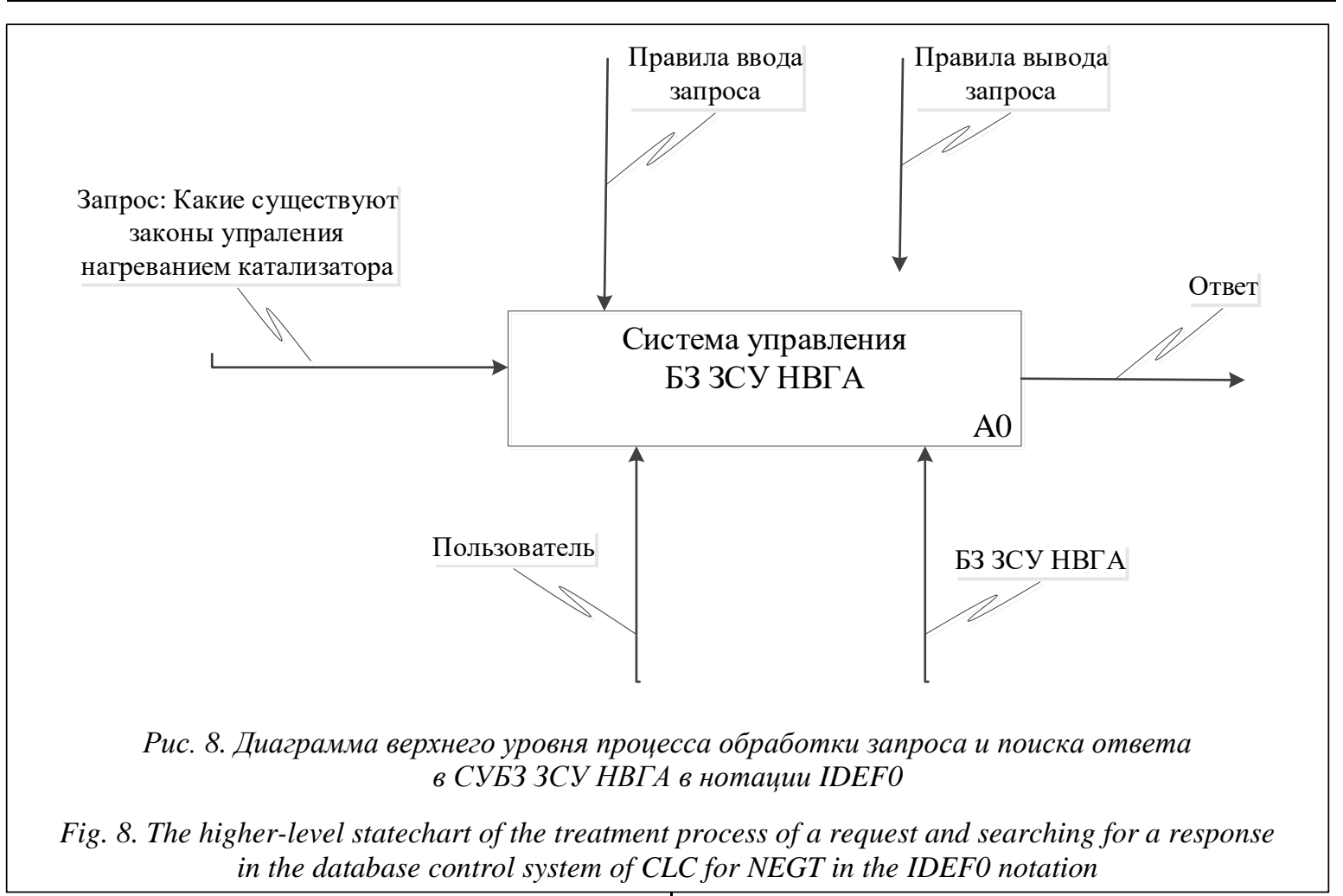
СУБЗ:

Общие функциональные требования к

- хранить, упорядочивать, классифицировать знания о предметных областях, связанных с ЗСУ НВГА, в том числе о данных элементов, схемотехнических, конструкторских и других проектно-производственных решений;

- предоставлять информацию об инновационных и нестандартных проектных решениях, связанных со структурой ЗСУ НВГА и ее отдельных элементов.

\section{Алгоритмы работы СУБЗ ЗСУ НВГА}

СУБЗ ЗСУ НВГА имеет двухуровневую структуру:

- хранилище знаний (Б3), а именно, внутренний каталог, к которому пользователь не имеет доступа для обеспечения сохранности, достоверности и исключения несанкционированных правок;

- модули обработки запросов и вывода результатов.

СУБЗ ЗСУ НВГА должна работать следующим образом: после отправки запроса пользователем в систему из БЗ поступает соответствующий ответ; если ответ на запрос не найден, пользователь увидит пустой список, а система предложит ввести повторный запрос, после чего происходят обработка запроса и выдача результата.

Рассмотрим алгоритм работы СУБЗ ЗСУ НВГА на примере запроса «Какие существуют законы управления нагреванием катализатора». На рисунке 8 представлен процесс обработки указанного запроса и поиска ответа на него. Вводимый запрос должен удовлетворять правилам ввода запроса.

На рисунке 9 изображена трансформация запроса в модуль обработки. В этом модуле срабатывает правило «если вводимый запрос

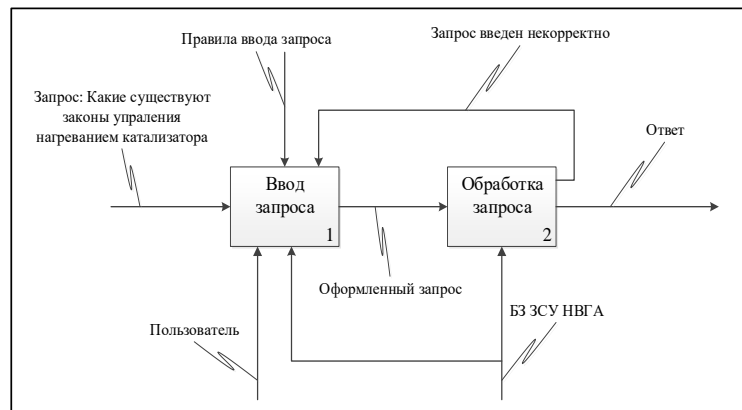

Рис. 9. Контекстная диаграмма 1-го уровня проиесса обработки запроса и поиска ответа в СУБЗ ЗСУ НВГА в нотаџчи IDEFO

Fig. 9. The context diagram of the first level of the treatment process of a request and searching for a response in the database control system of CLC for NEGT in the IDEFO notation 
не удовлетворяет правилам ввода запроса, то программа попросит пользователя повторно ввести запрос».

Далее происходят поиск и выдача результата в СУБЗ ЗСУ НВГА. Если ответ не найден, то список на экране пуст, в противном случае программа показывает список ответов «Релейный закон», «Закон ПИД-регулятора» и др. (см. http://www.swsys.ru/uploaded/image/2020-4/ 2020-4-dop/6.jpg).

\section{Возможные запросы для СУБЗ ЗСУ НВГА}

Представим примерный перечень возможных запросов к БЗ ЗСУ НВГА от проектировщиков.

Схемотехнический этап ЗСУ НВГА:

- экологические стандарты «Евро» для автотранспорта;

- параметры токсичных веществ;

- структура;

- функциональная схема;

- передаточные функции звеньев;

- математические модели катализатора;

- принципиальная схема микроконтроллера;

- законы управления катализатором;

- конструкторские решения ЗСУ НВГА на этапе конструирования;

- 3D-модель конструкции катализатора;

- 2D-модель элементов катализатора;

- таблицы характеристик катализаторов, микроконтроллеров, ГЛОНАСС, датчиков ВГА, датчиков температуры, индикаторов ВГА.

Этап технологической подготовки производства:

- вероятностные характеристики переходов при выполнении технологических и производственных процедур;

- выбор элементной базы для серийно способных изделий;

- замена импортных компонентов на российские аналоги;

- контроль соответствия Gerber-файлов технологии линии автоматического монтажа печатных плат.

\section{Интерфейс пользователя Б3}

В интерфейсе пользователя (см. http://www. swsys.ru/uploaded/image/2020-4/2020-4-dop/7. jpg) имеются поля:

- ввода запроса;

- списка запросов;

- индикации ответов в текстовом виде;

- ввода текста для поиска,

а также кнопки поиска ответа и визуализации текста.

На рисунке (см. http://www.swsys.ru/uploaded/image/2020-4/2020-4-dop/7.jpg) представлены ответы на запрос конструктора.

На рисунке (см. http://www.swsys.ru/uploaded/image/2020-4/2020-4-dop/8.jpg) показан выбор основных элементов системы: окно «Ввод запроса» дает возможность пользователю быстро найти нужные элементы по параметрам в полях «Выбор катализатора», «Выбор модуля ГЛОНАСС», «Выбор контроллера», «Выбор датчика ВВГ», «Выбор термодатчика», «Выбор GSM», «Выбор индикатора ВВГ»; кнопка «Анализ» служит для оценки вариантов выбора элементов системы.

\section{Заключение}

Таким образом, предложенный подход к построению и применению БЗ на конкретных процедурах этапов схемотехнического, конструкторского проектирования, технологической подготовки производства сводится к разработке БЗ и СУБЗ - необходимой составляющей инструментария проектировщика. Использование онтологического инжиниринга перспективно как в построении, так и в применении предложенных программных средств.

Полученные результаты подтверждают перспективность распространения предложенных разработок БЗ на широкий класс инновационных объектов, которое сводится к расширению приведенной СУБЗ на соответствующие проблемно-ориентированные языки и другие составляющие адаптации к конкретному объекту проектирования.

\section{Лumepamypa}

1. Hayes-Roth F., Waterman D.A., Lenat D. Building Expert Systems. Addison-Wesley Publ., 1983, $444 \mathrm{p}$.

2. Green C., Luckham D., Balzer R., Cheatham T., Rich C. Report on a knowledge-based software assistant. In: Readings in Artificial Intelligence and Software Engineering. Morgan Kaufmann Publ., 1986, pp. $377-428$. DOI: 10.1016/B978-0-934613-12-5.50034-3.

3. Гаврилова Т.А. Базы знаний интеллектуальных систем. СПб: Питер, 2001. 384 с. 
4. Старцева Е.Б. Этапы построения модульной структуры базы знаний экспертной системы на основе системного подхода // Вестн. УГАТУ. 2006. № 2. С. 178-181.

5. Наумов А.Н., Вендров А.М., Иванов В.К., Москаев А.В. Системы управления базами данных и знаний. М.: Финансы и статистика, 1991. 352 с.

6. Сольницев Р.И., Коршунов Г.И. Системы управления «природа-техногеника». СПб: Политехника, 2012. 205 с.

7. Сольницев Р.И., Коршунов Г.И., Петрушевская А.А., Параничев А.В. Киберфизические системы в экологической безопасности и геомониторинге автотранспорта. СПб: Изд-во СПбГЭТУ «ЛЭТИ», 2019. $200 \mathrm{c}$.

8. Коршунов Г.И., Петрушевская А.А., Чуписов А.Е. Совершенствование технологических процессов при производстве и испытаниях инновационной электроники // Вопросы радиоэлектроники. 2017. № 10. С. $15-19$.

9. Gruninger M., Atefi K., Fox S.M. Ontologies to support process integration in enterprise engineering. Computational and Mathematical Organization Theory, 2000, vol. 6, pp. 381-394.

10. Маклаков С.В. Моделирование бизнес-процессов с BPwin 4.0. М.: Диалог-МИФИ, 2002.224 с.

11. Бабанов А.М. Технология разработки программного обеспечения: структурный подход. Томск: Изд-во ТГУ, 2006. 157 с.

12. Protégé. URL: http://protege.stanford.edu (дата обращения: 15.04.2020).

13. DAML Ontology Library. URL: http://www.daml.org/ontologies (дата обращения: 15.04.2020).

14. Resource Description Framework (RDF). URL: http://www.w3.org/RDF/ (дата обращения: 15.04.2020).

\section{Issues of construction and application of knowledge basis in designing and production of innovative objects}

R.I. Solnitsev ${ }^{1}$, Dr.Sc. (Engineering), Professor, remira70@mail.ru

G.I. Korshunov ${ }^{2}$, Dr.Sc. (Engineering), Professor, kgi@pantes.ru

Do Xuan Cho ${ }^{3}$, Ph.D. (Engineering), chodx@ptit.edu.vn

Do Hai Kuan ${ }^{1}$, Graduate Student, quan221212@gmail.com

${ }^{1}$ St. Petersburg Electrotechnical University "LETI", St. Petersburg, 197376, Russian Federation

${ }^{2}$ St. Petersburg State University of Aerospace Instrumentation, St. Petersburg, 190000, Russian Federation

${ }^{3}$ Vietnam Posts and Telecommunications Institute, Ha Noi, Vietnam

Abstract. The paper discusses the principles of building and applying a knowledge base (KB) for innovative objects. The paper describes the construction and application of knowledge base in designing and production on the basis of an example of a closed control system for the neutralization of exhaust gases of transports, which is an innovative object in the field of the functioning of Nature-Technogenics control system. The authors substantiate the choice of a closed control system for the neutralization of exhaust gases of transports as a standard for innovative design and production facilities.

The design and production process of this object will be built on a knowledge base, which includes the development of principle diagrams and circuits, structural solutions and documentation, software, production, assembling, and adjusting of prototypes.

In the knowledge base, there must be data and knowledge to design and produce research objects. So, for the circuit design stage, an electrical scheme is introduced for a closed control system for the neutralization of exhaust gases of transports, which developed by PCAD design tools in accordance with the established standards stored in the knowledge base. CAD and PPAS tools for the next steps will ensure the calculation of IPC standards also through the knowledge base, which includes the development of design solutions and documentation for the production of printed circuit boards and other elements, and PCBA verification. The development of project documentation at subsequent design stages is also based on a knowledge base. At the same time, the structure fragments stored in the knowledge base are used for new design solutions and the production of design documentation. 
The paper also proposes a knowledge base management system, which will allow developers to use UML notation to provide support its, receive answers from requests of "ordinary" design engineer (circuit engineers, designers, technologists, testers), experts will ensure the input and control of knowledge.

The user interface is also presented, in which there are fields "enter requests", "show answers", as well as control and visualization operators. The application of the developed knowledge base for the procedures into the circuit design, architectural design stages, and technological preparation for the production is shown in examples.

The knowledgebase application for specific production and design sequences is made on the basis of the user's problem-oriented language when creating requests and answers.

Keywords: closed-loop control, innovative objects, knowledgebase, knowledgebase management system, catalytic converter, design, and production.

\section{References}

1. Hayes-Roth F., Waterman D.A., Lenat D. Building Expert Systems. Addison-Wesley Publ., 1983, $444 \mathrm{p}$.

2. Green C., Luckham D., Balzer R., Cheatham T., Rich C. Report on a knowledge-based software assistant. In: Readings in Artificial Intelligence and Software Engineering. Morgan Kaufmann Publ., 1986, pp. 377-428. DOI: 10.1016/B978-0-934613-12-5.50034-3 (in Russ.).

3. Gavrilova T.A. Knowledge Bases of Intelligent Systems. St. Petersburg, 2001, 384 p.

4. Startseva E.B. Stages of building a modular structure of the knowledge base of an expert system based on a systematic approach. Vestn. UGATU, 2006, no. 2, pp. 178-181 (in Russ.).

5. Naumov A.N., Vendrov A.M., Ivanov V.K., Moskaev A.V. Database and Knowledge Management Systems. Moscow, 1991, 352 p. (in Russ.).

6. Solnitsev R.I., Korshunov G.I. The Control System "Nature-Technogenics". St. Petersburg, 2012, 205 p. (in Russ.).

7. Solnitsev R.I., Korshunov G.I., Petrushevskaya A.A., Paranichev A.V. Cyberphysical Systems in Environmental Safety and Geomonitoring of Vehicles. St. Petersburg, 2019, 200 p. (in Russ.).

8. Korshunov G.I., Petrushevskaya A.A., Chupisov A.E. Improvement of technological processes in the production and testing of innovative electronics. Issues of Radio Electronics, 2017, no. 10, pp. 15-19 (in Russ.).

9. Gruninger M., Atefi K., Fox S.M. Ontologies to support process integration in enterprise engineering. Computational and Mathematical Organization Theory, 2000, vol. 6, pp. 381-394.

10. Maklakov S.V. Modeling Business Processes with BPwin 4.0. Moscow, 2002, 224 p. (in Russ.).

11. Babanov A.M. Technology of Software Development: The Structural Approach. Tomsk, 2006, 157 p. (in Russ.).

12. Protégé. Available at: http://protege.stanford.edu (accessed April 15, 2020)

13. DAML Ontology Library. Available at: http://www.daml.org/ontologies (accessed April 15, 2020).

14. Resource Description Framework $(R D F)$. Available at: http://www.w3.org/RDF/ (accessed April 15, 2020).

\section{Для цитирования}

Сольницев Р.И., Коршунов Г.И., До Суан Чо, До Хай Куан. Вопросы построения и применения базы знаний в проектировании и производстве инновационных объектов / / Программные продукты и системы. 2020. Т. 33. № 4. C. 564-572. DOI: 10.15827/0236-235X.132.564-572.

\section{For citation}

Solnitsev R.I., Korshunov G.I., Do Xuan Cho, Do Hai Kuan. Issues of construction and application of knowledge basis in designing and production of innovative objects. Software \& Systems, 2020, vol. 33, no. 4, pp. 564-572 (in Russ.). DOI: 10.15827/0236-235X.132.564-572. 